Früher Insulineinsatz bei Typ-2-Diabetes

\section{Patientenzufriedenheit und Lebensqualität steigen}

- In der Behandlung bei Diabetes Typ 2 zeichnet sich ein Paradigmenwechsel ab. Statt die Insulintherapie wie bisher möglichst lange hinauszuzögern, werden Insuline immer öfter frühzeitig eingesetzt. Dabei sind Patienten bei Verwendung eines Insulinanalogons im Vergleich zu NPH-Insulin mit der Therapie zufriedener, wie eine Beobachtungsstudie ergeben hat.

Viele Patienten stehen einer Insulintherapie zunächst ängstlich gegenüber. Bedenken vor Hypoglykämien, Gewichtszunahme oder Abhängigkeit von Insulin sind groß. Prof. Norbert Hermanns, Bad Mergentheim, wies darauf hin, dass die Ängste der Patienten stark von der aktuellen Therapieform abhängen und sich zwischen insulinnaiven und insulintherapierten Patienten unterscheiden. Um die Therapiezufriedenheit und Lebensqualität einer neu initiierten Insulintherapie zu untersuchen, wurde die prospektive Beobachtungsstudie DE-LIGHT konzipiert. An ihr nahmen 8125 Patienten mit oralen Antidiabetika teil, die erstmals zusätzlich Insulin erhielten. Zwei Drittel der Diabetiker erhielten das lang wirksame Insulindetemir $\left(\right.$ Levemir $\left.^{\circledR}\right)$, ein Drittel wurde mit NPH-Insulin behandelt.

Die Therapiezufriedenheit, gemessen mit dem diabetesspezifischen DTSQs-Score, nahm in beiden Studiengruppen zu. In der Detemirgruppe stieg der Wert von 22,8 auf 27,2 Punkte, was eine signifikant stärkere Verbesserung bedeutet als unter NPHInsulin mit einem Anstieg von 22,1 auf 25,9 Punkte. Auch die Lebensqualität besserte sich mit dem Insulinanalogon in mehreren Scores (ADDQuol, Euro Quol 5D) signifikant stärker.

Dr. Marcel Kaiser, Frankfurt am Main, wies darauf hin, dass die (sekundären) klinischen Zielparameter das vorteilhafte Profil von Insulindetemir bestätigten. In der Studie sank innerhalb von sechs Monaten der $\mathrm{HbA}_{1 \mathrm{c}}$-Wert um 1,13\% (Detemir) bzw um 1,01\% (NPH-Insulin) auf jeweils 7,3\%. Unter dem neuen Verzögerungsinsulin war eine Abnahme des Nüchternblutzuckers um 44,8 mg/dl auf 130,6 $\mathrm{mg} / \mathrm{dl}$ zu verzeichnen, signifikant stärker als in der Kontrollgruppe (um 38,5 mg/dl auf 132,1 mg/dl).

Hypoglykämien traten unter dem Insulinanalogon mit einer Rate von 0,58 gegenüber 0,80 bzw. bei nächtlichen Hypoglykämien mit 0,09 gegenüber 0,21 signifikant seltener auf. Das Körpergewicht sank unter Insulindetemir um 1,16 kg, mit NPH-Insulin nur um $0,4 \mathrm{~kg}$.

\section{Zulassungserweiterung für Gliptin}

Der DPP-4-Hemmer Sitagliptin (z.B. Xelevia ${ }^{\circledR}$ ) ist nun auch für die Monotherapie bei Metforminunverträglichkeit oder -kontraindikationen sowie für die Dreifachtherapie mit Metformin und einem Glitazon zugelassen. Für die Kombitherapie steht das Gliptin auch als Fixkombination mit Metformin (z.B. Velmetia ${ }^{\circledR}$ ) zur Verfügung. Berlin Chemie

\section{Umstellung auf Plasmakalibrierung}

Roche Diagnostics hat seine Blutzuckermesssysteme seit September auf Plasmakalibrierung umgestellt. Die neuen Teststreifen unterscheiden sich in der Handhabung nicht von den bisherigen vollblutkalibrierten Streifen. Da die Glukosekonzentration im Blutplasma höher ist als im Vollblut, zeigen die Messsysteme bei gleicher Stoffwechsellage mit den neuen Streifen ca. 10-15\% höhere Messwerte an. Roche Diagnostics

\section{Diabetesmanagement via Handy}

Ein innovatives telemedizinisches Blutzuckermonitoring- und Diabetesmanagement-System bietet das Unternehmen Bodytel ${ }^{\mathrm{TM}}$ mit dem Namen GlucoTel an. Mit dem System werden die Dateneingabe und die Verwaltung eines Diabetestagebuchs in einem zugehörigen Onlineportal besonders einfach. Das System richtet sich v.a. an junge und mobile Menschen, die die manuelle Dateneintragung umgehen möchten. Sein Herzstück ist eine kostenlose Handy-Software, BodyTel Mobile, mit deren Hilfe BZMesswerte an die Datenbank geleitet werden. Die Übertragung zwischen Messgerät und Handy erfolgt automatisch via Bluetooth über bis zu zehn Meter. Durch den bei eingeschaltetem Handy ständigen Informationsfluss entsteht ein lückenloses Onlinetagebuch. Zugriff haben außer dem Patienten über Kennwort autorisierte Personen. Ist das Handy aus, speichert das Messgerät bis zu 100 Werte und überträgt sie später mit. Via Internet können Informationen wie Mahlzeiten und Sport integriert werden. Bei Über- oder Unterschreitung definierter Grenzwerte wird ein Alarm per SMS, Fax oder E-Mail an Vertrauenspersonen schickt. Weitere Infos unter:

Bodytel

Kurz notiert 